\title{
Experimental investigation of the response of an amorphous silicon EPID to intensity modulated radiotherapy beams
}

\author{
Peter B. Greer ${ }^{\text {a) }}$ \\ Calvary Mater Newcastle Hospital, Newcastle, New South Wales, Australia and University of Newcastle, \\ New South Wales, Australia \\ Philip Vial and Lyn Oliver \\ Royal North Shore Hospital, Sydney, Australia and Institute of Medical Physics, University of Sydney, \\ Sydney, Australia \\ Clive Baldock \\ Institute of Medical Physics, University of Sydney, Sydney, Australia
}

(Received 22 March 2007; revised 10 August 2007; accepted for publication 29 August 2007; published 24 October 2007)

\begin{abstract}
The aim of this work was to experimentally determine the difference in response of an amorphous silicon $(a-\mathrm{Si})$ electronic portal imaging device (EPID) to the open and multileaf collimator (MLC) transmitted beam components of intensity modulated radiation therapy (IMRT) beams. EPID dose response curves were measured for open and MLC transmitted $\left(\mathrm{MLC}_{\mathrm{tr}}\right) 10 \times 10 \mathrm{~cm}^{2}$ beams at central axis and with off axis distance using a shifting field technique. The EPID signal was obtained by replacing the flood-field correction with a pixel sensitivity variation matrix correction. This signal, which includes energy-dependent response, was then compared to ion-chamber measurements. An EPID calibration method to remove the effect of beam energy variations on EPID response was developed for IMRT beams. This method uses the component of open and $\mathrm{MLC}_{\mathrm{tr}}$ fluence to an EPID pixel calculated from the MLC delivery file and applies separate radially dependent calibration factors for each component. The calibration procedure does not correct for scatter differences between ion chamber in water measurements and EPID response; these must be accounted for separately with a kernel-based approach or similar method. The EPID response at central axis for the open beam was found to be $1.28 \pm 0.03$ of the response for the $\mathrm{MLC}_{\mathrm{tr}}$ beam, with the ratio increasing to 1.39 at $12.5 \mathrm{~cm}$ off axis. The EPID response to $\mathrm{MLC}_{\mathrm{tr}}$ radiation did not change with off-axis distance. Filtering the beam with copper plates to reduce the beam energy difference between open and $\mathrm{MLC}_{\mathrm{tr}}$ beams was investigated; however, these were not effective at reducing EPID response differences. The change in EPID response for uniform sliding window IMRT beams with $\mathrm{MLC}_{\text {tr }}$ dose components from $0.3 \%$ to $69 \%$ was predicted to within $2.3 \%$ using the separate EPID response calibration factors for each dose component. A clinical IMRT image calibrated with this method differed by nearly $30 \%$ in high $\mathrm{MLC}_{\mathrm{tr}}$ regions from an image calibrated with an open beam calibration factor only. Accounting for the difference in EPID response to open and $\mathrm{MLC}_{\mathrm{tr}}$ radiation should improve IMRT dosimetry with $a$-Si EPIDs. (C) 2007 American Association of Physicists in Medicine. [DOI: 10.1118/1.2789406]
\end{abstract}

Key words: amorphous silicon, EPID dosimetry, energy response, intensity modulated

\section{INTRODUCTION}

The use and development of amorphous silicon electronic portal imaging devices ( $a$-Si EPIDs) for verification of delivered intensity modulated therapy (IMRT) is increasing. These devices have many attractive features for dosimetry due to their integration with the linear accelerator and imaging software. There is minimal set-up time involved, and the measurements obtained have high resolution. A full twodimensional record of the beam is obtained. The $a$-Si EPID consists of a metal plate for photon buildup, a gadolinium oxysulfide phosphor layer to produce visible light, and a photodiode detector array. The dosimetric properties of these devices have therefore received some interest. The response of $a$-Si EPIDs has been found to be linear, ${ }^{1-3}$ although some investigations have found changes in response at low doses and an image lag effect, which increases the EPID response with increasing dose. ${ }^{4-7}$ Backscatter from components of the EPID support arm downstream from the detector have also been found to influence the signal by up to $5 \% .^{8}$ However central axis long-term reproducibility measurements have been promising. ${ }^{9}$ These investigations have spurred development of methods to verify IMRT beams with these devices. ${ }^{10-16}$

A problem for these devices however is that they exhibit a beam energy-dependent response. Energy variation occurs in a typical IMRT field due to dose being delivered from both open beam and multileaf collimator (MLC) transmitted components. McCurdy et al. ${ }^{17}$ investigated with Monte Carlo modeling the central axis response of an $a$-Si EPID to monoenergetic incident photon beam energy. They found that the EPID was more sensitive at low energies due to the ga- 
dolinium oxysulfide phosphor layer. This has a high atomic number and therefore is more sensitive to low energy photons, particularly below $1 \mathrm{MeV}$. Kirkby et al. ${ }^{18}$ measured central axis EPID response to open fields and beams attenuated by compensators. They found that the EPID response was reduced for the compensated beams by up to $8 \%$ relative to the open beam, and a $0.7 \mathrm{~cm}$ copper filter plate reduced this difference to less than $4 \%$. We have previously found experimentally that the EPID response to off axis radiation (which has a greater low energy component than central axis) was increased by up to $13 \%$ at $15 \mathrm{~cm}$ off axis relative to the central axis. ${ }^{19}$ Parent et al. ${ }^{20}$ measured and Monte Carlo modeled an $a$-Si EPID and found that the response was increased by up to $29 \%$ at off-axis distances relative to central axis. EPID images are divided by a flood-field (FF) calibration image to correct for differences in pixel gains. When a FF image is acquired there is an energy variation across the beam. As the EPID has an energy-dependent response this results in an EPID response variation across the image. Therefore subsequent open-field images that are acquired and then divided by the FF have this energy-dependent response removed. The off-axis dependence of EPID response is then not usually apparent, provided the image is acquired under similar conditions to the FF, i.e., an open beam without a patient or phantom present and with the same EPID position.

These earlier works suggest that the response of the $a-\mathrm{Si}$ EPID to MLC transmitted radiation will be different from the response to open beams. The MLC transmitted beam will be heavily filtered with fewer low energy photons present compared to an open beam. While the MLC transmission is small at $\sim 1.5 \%$ of an open $10 \times 10 \mathrm{~cm}^{2}$ beam for modern MLC designs, ${ }^{21}$ during IMRT delivery a dose point may be irradiated by MLC-transmitted radiation for most of the beam on time. Therefore the dose delivered to a point may have a significant MLC transmission component. A difference in EPID response to the open and MLC-transmitted components could result in errors in EPID dosimetry for IMRT beams. By quantifying the difference in response to open and MLC beams, correction methods could be developed for EPID images of IMRT beams. Nicolini et al. ${ }^{15}$ measured calibration curves at central axis for both open and MLCtransmitted fields by comparison to ion-chamber measurements. They measured these for varying field size and with added buildup layers to give water equivalent EPID depths of $0.8,1.5,3.8$, and $10 \mathrm{~cm}$. Their results with doses delivered through the MLC up to $4 \mathrm{cGy}$ suggest a response to open beam of $\sim 2 \%$ greater than to MLC-transmitted beam for no added buildup on the EPID and a $10 \times 10 \mathrm{~cm}^{2}$ field size. EPID images of IMRT fields were calibrated to dose for each field segment separately using calibration factors for each segment field size and the component of dose for open and MLC-transmitted radiation calculated from the MLC delivery file. In this work we incorporate the difference in EPID response between open and MLC-transmitted beams into a more general EPID calibration method that determines the response for each pixel in the image and includes off-axis response effects. Li et al. ${ }^{22}$ used Monte Carlo methods to determine EPID dose-deposition kernels for open and MLCtransmitted radiation as a function of radial distance. They found the ratio of the EPID response for the open beam relative to the $\mathrm{MLC}_{\mathrm{tr}}$ beam at central axis was $\sim 1.3$. They convolved incident fluence derived from a commercial treatment planning system for the open and $\mathrm{MLC}_{\mathrm{tr}}$ dose components of an IMRT beam with these kernels to predict dosimetric EPID images.

The aim of this work is to experimentally determine the difference in response of an $a$-Si EPID to the open and MLC-transmitted beam components of intensity modulated beams and determine whether filtering of the beam with copper layers will reduce this response difference. The EPID central axis and off-axis energy-dependent responses are measured for open and MLC-transmitted $10 \times 10 \mathrm{~cm}^{2}$ beams, as well as with copper sheets present to filter the low energy beam components. With uniform sliding window irradiations, EPID response changes are compared to predictions using the components of open and MLC-transmitted radiation and the measured calibration factors. A previously developed EPID calibration procedure is extended to IMRT beams. Separate radially dependent EPID response (relative to ion-chamber) correction matrices are applied to the open and MLC-transmitted components of a clinical IMRT field, and the results compared with the standard method of applying an open field calibration factor to both dose components.

\section{METHODS AND MATERIALS}

\section{II.A. Materials}

All measurements were performed on a 21EX accelerator (Varian, Palo Alto, CA), with nominal 6 MV energy photon beams, and a Millenium 120 leaf MLC model. The dose rate was $300 \mathrm{MU} / \mathrm{min}$ for all measurements. The MLC leaves are made of tungsten with a leaf width of $0.5 \mathrm{~cm}$ (defined at $100 \mathrm{~cm}$ from the source) for the central $20 \mathrm{~cm}$ of the field and $1.0 \mathrm{~cm}$ width outside this and a thickness of $6 \mathrm{~cm}$ in the beam direction. The leaves move in the cross-plane direction with the collimator positioned at $0^{\circ}$ and are parallel to the $\mathrm{X}$-collimator jaws. The secondary collimator jaws are positioned above the MLC, with the X-jaws being lower and the Y-jaws upper. The Y-jaws have the capability to move up to $10 \mathrm{~cm}$ over the central axis, whereas the X-jaws can only move up to $2 \mathrm{~cm}$ over the axis.

The EPID was the aS500 amorphous silicon detector. This detector is $40 \times 30 \mathrm{~cm}^{2}$ in size, with a matrix of 512 pixels in the cross-plane direction and 384 pixels in the inplane direction. Each pixel has a square pitch of $0.784 \mathrm{~mm}$. Unless otherwise stated the EPID was positioned with the surface of the detector at $105 \mathrm{~cm}$ from the source. The EPID vertical positioning was calibrated so that the surface of the detector sandwich corresponds to the displayed vertical position. No additional buildup was used on the EPID for these measurements. The inherent buildup of the EPID is equivalent to approximately $0.9 \mathrm{~cm}$ water equivalent. ${ }^{3}$ The EPID was operated with continuous frame acquisition during beam delivery. The number of reset frames before image acquisi- 
tion was zero. The reset frame every 64 frames was removed by a software update. The PV Client software version was 6.1.13 with IAS2 software version 6.1.11 and the detector IDU-11 model.

\section{II.B. EPID response to open and MLC-transmitted beams}

The open beam EPID dose response calibration curve was measured at the accelerator central axis with a $10 \times 10 \mathrm{~cm}^{2}$ field size. EPID images were acquired for monitor unit (MU) settings of $5,10,20,30,40,50,75,100$, and 200. At least two images were acquired for each MU setting. The EPID response was obtained by first removing the automatically applied FF correction (by multiplication of the FF-corrected image by the FF) and then correcting the EPID image with a previously derived pixel sensitivity matrix (PSM) that corrects for difference in pixel gains. ${ }^{19}$ This yields an image that includes EPID response variations due to energy variation in the beam. The EPID integrated response for each field was then obtained from the mean pixel value in a $9 \times 9$ pixel region at the center of the frame-averaged image multiplied by the number of frames acquired. The dose from these fields was determined with a $0.6 \mathrm{cc}$ ion chamber placed in a solid water phantom of thickness $14.5 \mathrm{~cm}$ at a depth of $1.5 \mathrm{~cm}$ and a source to surface distance $(\mathrm{SSD})$ of $100 \mathrm{~cm}$. The EPID measurements were made at $105 \mathrm{~cm}$ distance due to positioning constraints of the EPID arm and the EPID signal was scaled to the $100 \mathrm{~cm}$ plane using the inverse square law. The ion-chamber measurements were made at $100 \mathrm{~cm}$ SSD as this is the calibration condition for the accelerator and enabled conversion of readings to dose.

The EPID response to the MLC-transmitted $\left(\mathrm{MLC}_{\mathrm{tr}}\right)$ beam was then measured with the multileaf collimator completely closed and blocking the $10 \times 10 \mathrm{~cm}^{2}$ field defined by the secondary collimator jaws. The collimator was set to $0^{\circ}$ with the MLC leaves oriented in the cross-plane direction. A MLC field was set with the MLC leaf banks junctioned at $7 \mathrm{~cm}$ from the central axis. This field completely blocked a symmetric $10 \times 10 \mathrm{~cm}^{2}$ field formed by the secondary collimators without introducing the MLC carriage into the field. Large MU settings from 200 to 3333 were delivered due to the low dose transmitted through the MLC. The EPID response was obtained as above but in this case a larger 50 $\times 19$ pixel region was used due to the variation in the EPID signal due to interleaf leakage effects. This size region approximates the area under the MLC leaves measured by the ionization chamber.

To determine the dose delivered for these fields the MLC transmission factor was measured with the ion chamber in solid water using the experimental setup described in the previous paragraphs. Three separate readings were obtained and averaged. The long axis of the ion chamber was perpendicular to the direction of leaf motion. The uncertainties in the readings were estimated by moving the chamber $2 \mathrm{~mm}$ in each direction (perpendicular to leaf motion) and acquiring further readings. The experiment was also repeated on two separate occasions and the results compared. The dose deliv- ered for the $\mathrm{MLC}_{\mathrm{tr}}$ beams was then obtained from the MU settings and the MLC transmission factor. The dose could also have been determined directly from ion-chamber readings for each MU setting; however, we have previously verified the linearity of the linear accelerator output with MU setting, and therefore this approach was more efficient.

These measurements were then also repeated at $12.5 \mathrm{~cm}$ off axis. To maintain the MLC blocking of the field defined by the secondary jaws with the $0.5 \mathrm{~cm}$ width MLC leaves rather than introducing the $1 \mathrm{~cm}$ width leaves, the field was moved parallel to the direction of MLC leaf motion in the cross-plane direction (collimator=zero). As the X-collimator jaws are aligned with the MLC motion direction, these were used to define the field. The X-jaw can only be positioned at $2 \mathrm{~cm}$ over the central axis. The collimator settings used were -2 and $17 \mathrm{~cm}$, making a $15 \mathrm{~cm}$ field in the crossplane direction with $10 \mathrm{~cm}$ width maintained in the inplane direction. EPID images were then acquired for the open $15 \times 10 \mathrm{~cm}^{2}$ field for MU settings from 5 to 200, and for the MLC blocked field from 200 to 2000. The EPID energy-dependent response was then obtained as described above by removal of the FF and application of the pixel sensitivity correction matrix. The uncertainties in the EPID response were estimated by the standard deviation of repeated measurements.

Ion-chamber measurements were made off axis to determine the doses delivered to the EPID. Both ion-chamber and EPID sets of readings were adjusted to give the equivalent result for a $10 \times 10 \mathrm{~cm}^{2}$ field by using measured ion-chamber and EPID central axis output factors for a $15 \times 10 \mathrm{~cm}^{2}$ compared to a $10 \times 10 \mathrm{~cm}^{2}$ field. This reduced the ion-chamber measurements by $1 \%$ and the EPID readings by $2 \%$. While this introduces small uncertainties this was a compromise to ensure that the $0.5 \mathrm{~cm}$ width MLC leaves were always blocking the field. Introducing the $1 \mathrm{~cm}$ leaves could modify the MLC transmission measurements slightly due to change in the spacing of the interleaf leakage component. The results in this work, however, should apply to MLC systems entirely comprised of $1 \mathrm{~cm}$ thick leaves, as the thickness in the beam direction is the same.

\section{II.C. EPID response variation with off-axis distance}

The variation in EPID response to an MLC blocked beam with off-axis distance was investigated using a shifting field technique. To enable movement of the $10 \times 10 \mathrm{~cm}^{2}$ field to $15 \mathrm{~cm}$ off axis the collimator was rotated to $90^{\circ}$, which oriented the MLC leaves in the inplane direction. The Y-collimator jaws were then adjusted to move the 10 $\times 10 \mathrm{~cm}^{2}$ field in the cross-plane direction in $2.5 \mathrm{~cm}$ increments to $15 \mathrm{~cm}$ from the central axis (perpendicular to MLC leaf motion). The Y-jaws can move up to $10 \mathrm{~cm}$ over the central axis. Offsets of the field center greater than $15 \mathrm{~cm}$ are therefore not possible with the $10 \times 10 \mathrm{~cm}^{2}$ field size. The cross-plane direction was chosen to minimize variation in EPID backscattered radiation due to the support arm housing. EPID images were acquired at each off-axis distance of the MLC blocked beam. The MU set was 500. The energydependent EPID response at the center of each field was 
obtained for each distance as described above. At least two fields were recorded at each position and the results averaged.

To determine the dose for each field the ion chamber was positioned at the center of each field and oriented perpendicularly to the MLC leaf motion direction. The $0.6 \mathrm{cc}$ ion chamber was placed in a solid water phantom at a depth of $1.5 \mathrm{~cm}$ and a SSD of $98.5 \mathrm{~cm}$, with a further $12 \mathrm{~cm}$ of solid water to provide backscatter. The chamber was placed at the center of each offset $10 \times 10 \mathrm{~cm}^{2}$ blocked field using couch shifts and the dose relative to central axis recorded. The MU set was to 500 as above. Three readings were made at each position and averaged. As the ion chamber is $\sim 3 \mathrm{~cm}$ long, to ensure that details of the off-axis dose variation were not being missed, the measurements were also repeated with the chamber aligned parallel to the direction of MLC leaf motion. This, however, introduces greater variation in the measured dose due to intraleaf and interleaf MLC transmission. The dose was measured at $1 \mathrm{~cm}$ increments for the MLC blocked fields, with each measurement directly under the center of a MLC leaf. The perpendicular and parallel ionchamber measurements were compared with quadratic fits made to both sets of measurements to ensure that the (smoother) perpendicular orientation results could be utilized.

To determine the off-axis energy-dependent response of the EPID relative to ion chamber for the MLC blocked fields, quadratic fits were made to both EPID and ion-chamber data sets to smooth the effect of intraleaf and interleaf MLC transmission variation. The EPID off-axis energy-dependent response was then obtained by dividing the fitted EPID response by the fitted ion-chamber relative dose. The uncertainties in the measured response were estimated from the standard deviation of the differences between the measurements and the quadratic fit.

To verify that the open field EPID response was the same as previously measured ${ }^{19}$ the above was also repeated for open $10 \times 10 \mathrm{~cm}^{2}$ fields with the MLC retracted. Fields were offset in $1 \mathrm{~cm}$ increments to $5 \mathrm{~cm}$ off axis followed by $2.5 \mathrm{~cm}$ increments to $15 \mathrm{~cm}$ off axis. In this case EPID images were acquired of the open fields and processed as above to obtain the EPID response. Each irradiation was $100 \mathrm{MU}$. Ion-chamber measurements of the dose relative to central axis were recorded, and the EPID response relative to ion chamber calculated from the ratio of EPID to ion-chamber measurements.

\section{II.D. Effect of copper filters on the EPID energy- dependent response}

To determine whether copper filtering would reduce the differences in beam energy for open and MLC-transmitted beams and hence the difference in EPID response, the central axis EPID response curve measurements for the open and $\mathrm{MLC}_{\mathrm{tr}}$ beam were repeated with 1.0 and $1.6 \mathrm{~cm}$ thick copper $\mathrm{Cu}$ ) sheets. Large thicknesses of copper were used, as the MLC-transmitted beam passes through a large thickness of tungsten, and therefore to filter the open beam in a similar
TABLE I. The predicted change in EPID response for uniform sliding window IMRT beams based on the measured calibration factors for open and MLC-transmitted dose components is compared with the measured change in EPID response.

\begin{tabular}{lccc}
\hline \hline $\begin{array}{l}\text { MLC- } \\
\begin{array}{l}\text { Transmission } \\
\text { dose fraction }\end{array}\end{array}$ & $\begin{array}{c}\text { Normalized } \\
\text { ratio EPID/ion } \\
\text { chamber }\end{array}$ & $\begin{array}{c}\text { Predicted } \\
\text { ratio } \\
\text { EPID/ion } \\
\text { chamber }\end{array}$ & $\begin{array}{c}\text { Predicted/ } \\
\text { Measured }\end{array}$ \\
\hline 0.003 & 1.000 & 1.000 & 1.000 \\
0.006 & 0.997 & 0.999 & 1.002 \\
0.03 & 0.981 & 0.994 & 1.013 \\
0.06 & 0.988 & 0.988 & 1.000 \\
0.11 & 0.979 & 0.978 & 0.998 \\
0.17 & 0.967 & 0.962 & 0.996 \\
0.43 & 0.924 & 0.907 & 0.982 \\
0.69 & 0.869 & 0.848 & 0.977 \\
\hline \hline
\end{tabular}

way will require thick filtration. These were placed at the shadow tray level of the accelerator at $\sim 60 \mathrm{~cm}$ from the source. Kirkby and Sloboda ${ }^{18}$ found that placing plates at this level was more effective at reducing EPID response differences to open and compensated beams. Due to their weight these plates could not be placed on the surface of the EPID. Copper plates would not be present during patient treatment but could potentially be used for pretreatment IMRT verification, provided their effect on the beam profile can be incorporated into the treatment planning system modeling. These measurements were then also repeated at $12.5 \mathrm{~cm}$ off axis as described in Section II B but with the copper plates present. Ion-chamber measurements were made both at central axis and off axis to determine the attenuation of the copper plates and hence the doses delivered to the EPID.

\section{II.E. Sliding window dynamic MLC beams}

The change in EPID response for uniform intensity dynamic MLC beams with varying components of open and $\mathrm{MLC}_{\mathrm{tr}}$ dose was investigated. The secondary collimators were set to a $10 \times 10 \mathrm{~cm}^{2}$ field with a uniform leaf gap sweeping across this aperture. The leaf gap and leaf open fraction were varied to give the fraction of beam-on time at central axis with the MLC blocking the central axis from 0.17 to 0.99 . This varied the dose component delivered through the MLC leaves from $0.3 \%$ (open beam) to $69 \%$, assuming $1.5 \%$ transmission through the leaves. The MLCtransmission dose fractions for these fields are shown in the first column of Table I. The dose for each field was measured at central axis with a $0.6 \mathrm{cc}$ ion chamber (NE 2571) at $1.5 \mathrm{~cm}$ depth in a $12 \mathrm{~cm}$ thick solid water slab at a distance of $100 \mathrm{~cm}$ to the surface of the phantom. EPID images were also acquired of each field. The EPID integrated response was obtained over a $1 \times 1 \mathrm{~cm}^{2}$ area at the center of the image. These images were acquired at a separate center by author Vial using the same type of EPID and linear accelerator. The absolute calibration factors would not be applicable to this EPID due to a variety of potential differences; how- 


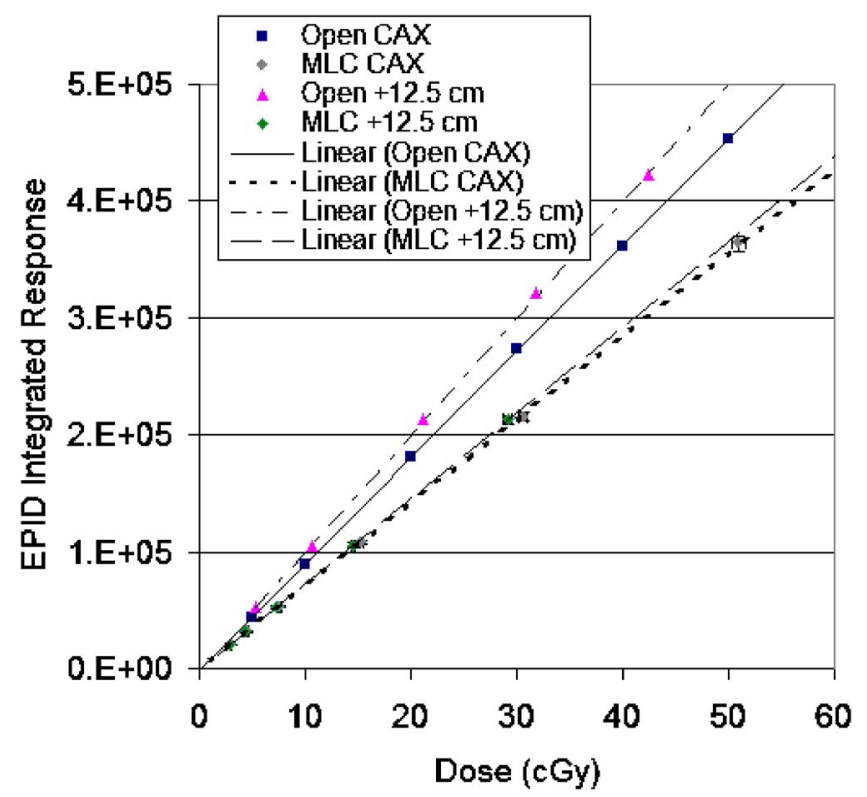

FIG. 1. EPID responses for open and MLC-transmitted radiation. These were measured at the central axis and at $12.5 \mathrm{~cm}$ off axis for open and MLC blocked $10 \times 10 \mathrm{~cm}^{2}$ fields.

ever, the ratio of EPID response to open and $\mathrm{MLC}_{\mathrm{tr}}$ beam would be expected to be the same. The $\mathrm{TPR}_{20,10}$ values for the $6 \mathrm{MV}$ beams for the two accelerators used in the study were 0.677 and 0.672 .

The measured reduction in EPID response relative to ion chamber with increasing $\mathrm{MLC}_{\mathrm{tr}}$ dose fraction for these fields is given in the second column of Table I. These are normalized to the "open" beam $\left(0.3 \%\right.$ MLC $\left._{\text {tr }}\right)$, where the EPID response is considered to be entirely due to open beam radiation. These were compared to a predicted change in EPID response $\left(\triangle \mathrm{EPI}_{C A X \text {,predicted }}^{\mathrm{resp}}\right)$ using separate calibration factors for the open and MLC transmitted dose components relative to the change in response with only open field calibration factors applied to both components. Equation (1) uses the components of open $\left(F^{\mathrm{op}}\right)$ and MLC transmitted dose [(1 $\left.\left.-F^{\mathrm{op}}\right) T\right]$ and the previously measured relative EPID responses to open and $\mathrm{MLC}_{\mathrm{tr}}$ beams. A response factor of 1 was used for the open component and 0.78 for the MLC $_{\text {tr }}$ component. The $0.78(=1 / 1.28)$ factor was obtained from the ratio of the slopes of the central axis EPID response curves for MLC-t and open beams (shown in Fig. 1). $T$ is the MLC transmission of $1.5 \%$.

$$
\begin{aligned}
\Delta \mathrm{EPI}_{C A X, \text { predicted }}^{\mathrm{resp}}= & \left(F^{\mathrm{op}}+0.78 \cdot\left(1-F^{\mathrm{op}}\right) T\right) /\left(F^{\mathrm{op}}+(1\right. \\
& \left.\left.-F^{\mathrm{op}}\right) T\right) .
\end{aligned}
$$

\section{II.F. Correction of energy-dependent response for IMRT beams}

A previously developed EPID response calibration method $^{19}$ was extended to IMRT beams by incorporating separate calibration factor matrices for the open and MLCtransmitted components of IMRT beams. The integrated EPID response $\left(\mathrm{EPI}_{i, j}^{\mathrm{resp}}\right)$ is obtained from the acquired image
$\left(\mathrm{EPI}_{i, j}^{\text {acquired }}\right)$ reported by the software, removal of the FF correction matrix $\left(\mathrm{FF}_{i, j} / \mathrm{FF}_{\text {mean }}\right)$, application of the pixel sensitivity matrix $\left(\mathrm{PSM}_{i, j}\right)$ and multiplication by the number of frames acquired (NF):

$$
\mathrm{EPI}_{i, j}^{\mathrm{resp}}=\mathrm{NF}\left(\mathrm{EPI}_{i, j}^{\text {acquired }} \cdot \mathrm{FF}_{i, j} / \mathrm{FF}_{\text {mean }}\right) / \mathrm{PSM}_{i, j}
$$

The method uses the concept of radially dependent EPID calibration factors for the open and $\mathrm{MLC}_{\mathrm{tr}}$ dose components based on the measured response for the $10 \times 10 \mathrm{~cm}^{2}$ fields. The radial calibration factors are determined from the measured central axis calibration factors (Section II B) divided by the radially dependent off-axis EPID response relative to ion chamber normalized at the central axis (Section II C). This approach calibrates the EPID to dose as measured with the ion chamber for a $10 \times 10 \mathrm{~cm}^{2}$ field size at central axis and corrects the image for changes in response due to energy variations in the beam. Field size response differences between EPID and ion chamber are not corrected. The radially dependent open field EPID response correction was mapped to a matrix in Cartesian coordinates at the EPID pixel resolution $\left(\mathrm{OAR}_{i, j}^{\mathrm{op}}\right)$. The $\mathrm{MLC}_{\mathrm{tr}}$ response off-axis correction was taken to be 1 for all EPID pixels $i, j$. This is due to the response of the EPID being approximately independent of radial distance as seen in Fig. 3 below. The EPID calibration matrices for the open $\left(C_{i, j}^{\mathrm{op}}\right)$ and $\mathrm{MLC}_{\mathrm{tr}}\left(C_{i, j}^{\mathrm{mlc}}\right)$ fluence components are then given by

$$
\begin{aligned}
& C_{i, j}^{\mathrm{op}}=C_{\mathrm{cax}}^{\mathrm{op}} / \mathrm{OAR}_{i, j}^{\mathrm{op}} \\
& C_{i, j}^{\mathrm{mlc}}=C_{\mathrm{cax}}^{\mathrm{mlc}} .
\end{aligned}
$$

$\mathrm{OAR}_{i, j}^{\mathrm{op}}$ was obtained from a quadratic fit to the data for offaxis energy-dependent response reported previously. ${ }^{19}$ The equation utilized was $y=k_{1} r^{2}+k_{2} r+k_{3}$, where $k_{1}=0.0005$, $k_{2}=0.0013$, and $k_{3}=0.996$. This is similar to a fourth order polynomial reported previously but gives a slightly improved fit to the measured data.

Only primary radiation (considering the radiation beam to be comprised of multiple pencil or ray beams from the source, with no scattered component) is considered with the approximation made that the dose to a given EPID pixel consists of an open beam component and an $\mathrm{MLC}_{\mathrm{tr}}$ component. These components are determined from the dynamic MLC treatment delivery file. Within this file a dose index is given for the dose fraction delivered from the leaf positions for a segment shape $s_{j-1}$ to the next leaf positions $s_{j}$. The leaf positions for each segment were determined, and these linearly interpolated into a further ten subsegment leaf positions. Each subsegment was assigned 1/10 of the dose index. All leaf positions were increased or "moved out" by $0.9 \mathrm{~mm}$ to account for the radiation transmission through the leaf tips. ${ }^{21}$ By accumulating the dose for all the segment shapes and dose indices in the file, a map of the fraction of the beam-on time or MU irradiated with the open beam is produced. This was then interpolated to a matrix at the EPID pixel scale at $100 \mathrm{~cm}$ from the source $\left(f_{i, j}^{\text {op }}\right)$. Therefore the fraction of the total dose delivered to a pixel due to the open beam component of the IMRT field is given by 


$$
F_{i, j}^{\mathrm{op}}=f_{i, j}^{\mathrm{op}} /\left(f_{i, j}^{\mathrm{op}}+\left(1-f_{i, j}^{\mathrm{op}}\right) T\right),
$$

where $T$ is the MLC transmission. The calibrated EPID image is produced from the radially dependent open and MLCtransmitted calibration matrices and the fraction of the dose delivered for each component of the IMRT beam to each pixel $i, j$ :

$$
\mathrm{EPI}_{i, j}^{\mathrm{cal}}=\mathrm{EPI}_{i, j}^{\mathrm{resp}} \cdot\left\lfloor\mathrm{F}_{i, j}^{\mathrm{op}} \mathrm{C}_{i, j}^{\mathrm{op}}+\left(1-\mathrm{F}_{i, j}^{\mathrm{op}}\right) \mathrm{C}_{i, j}^{\mathrm{mlc}}\right\rfloor .
$$

This produces an EPID image that is corrected for the difference in EPID response relative to ion chamber for open and MLC-transmitted components of an IMRT beam. The calibration procedure does not correct for differences between ion chamber in water measurements and EPID response due to scatter differences.

A clinical head and neck IMRT field with significant MLC-transmitted dose was calibrated using the above procedure. The central axis of the beam was first obtained using an open $10 \times 10 \mathrm{~cm}^{2}$ field image acquired immediately prior to the IMRT delivery. The $50 \%$ field edges in this image were determined using linear interpolation of pixel values and the midpoint determined for both in-plane and cross-plane directions. This yields the central axis pixel location for the IMRT image, and this was used to align the central axes of the fluence maps/calibration matrices and the EPID image. The calibrated image was also compared with the same field with the radially dependent open field calibration factor applied to both the open and $\mathrm{MLC}_{\mathrm{tr}}$ dose components. The latter method is similar to the standard EPID calibration procedure that uses a central axis calibration to dose and a FF correction that incorporates open field EPID energy-dependent response. The above comparison was also repeated for a clinical prostate field with little MLC leakage radiation.

\section{RESULTS}

\section{III.A. EPID response to open and MLC-transmitted beams}

Figure 1 shows the measured EPID response curve at the central axis for the open and MLC-transmitted $10 \times 10 \mathrm{~cm}^{2}$ beams. The ratio of EPID response for the open and $\mathrm{MLC}_{\mathrm{tr}}$ beam was $1.28 \pm 0.03$ obtained from the slopes of least squares linear fit to the data. Also shown is the result for $12.5 \mathrm{~cm}$ off axis. The open field response has increased; however, the $\mathrm{MLC}_{\mathrm{tr}}$ response is similar. Therefore the ratio of the open and $\mathrm{MLC}_{\text {tr }}$ response of the EPID increases to 1.39 at this distance.

\section{III.B. EPID response with off-axis distance}

The results for the relative dose at the center of each MLC-blocked $10 \times 10 \mathrm{~cm}^{2}$ field with off-axis distance are shown in Fig. 2. The results for the ion chamber oriented with its long axis both perpendicular and parallel to the MLC leaf-motion direction are shown. These results are similar, with more variability in the parallel chamber results. This is due to the greater susceptibility to interleaf leakage in this orientation. Quadratic function fits to each data set were within $0.6 \%$ of each other to $15 \mathrm{~cm}$ off axis.

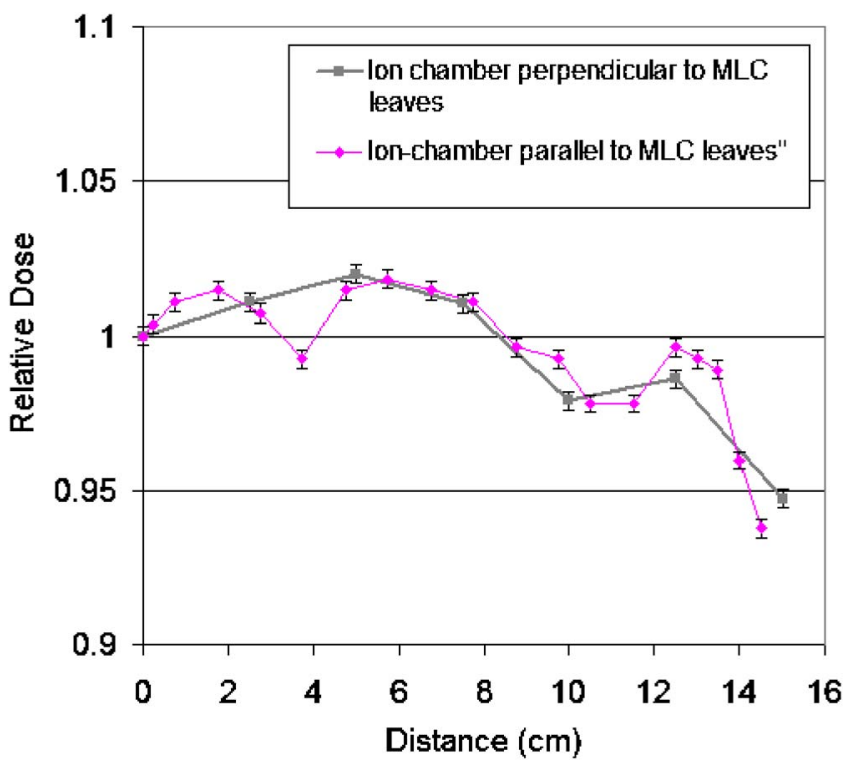

FIG. 2. Relative off-axis MLC-transmitted dose. This was measured at the center of $10 \times 10 \mathrm{~cm}^{2}$ fields completely blocked by the MLC with the ion chamber oriented perpendicular and parallel to the direction of MLC leaf motion.

Figure 3 shows the EPID response relative to central axis for the MLC-blocked $10 \times 10 \mathrm{~cm}^{2}$ fields compared to the measured ion-chamber dose for these fields. The EPID response is very similar to ion chamber for the $\mathrm{MLC}_{\mathrm{tr}}$ beam. The ratio of EPID response to ion chamber is also shown.

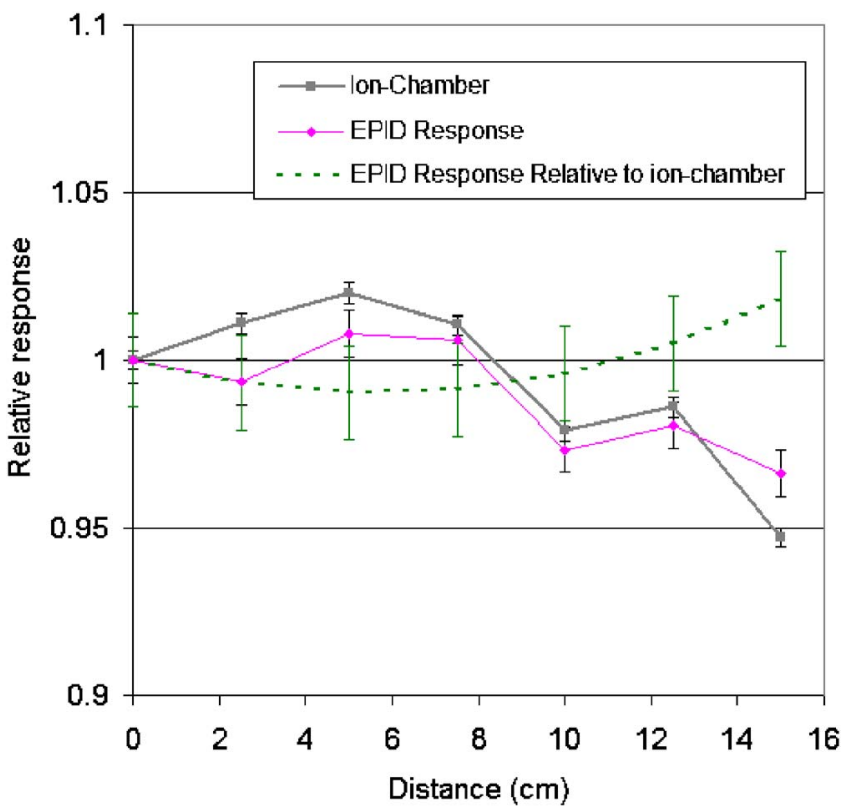

FIG. 3. EPID response for MLC-transmitted radiation with respect to offaxis distance relative to ion chamber. The EPID response for the 10 $\times 10 \mathrm{~cm}^{2}$ fields completely blocked by the MLC is compared to the measured ion-chamber dose for these fields. Data points are shown as well as a quadratic curve fit to the response of the EPID relative to the ion-chamber data. 


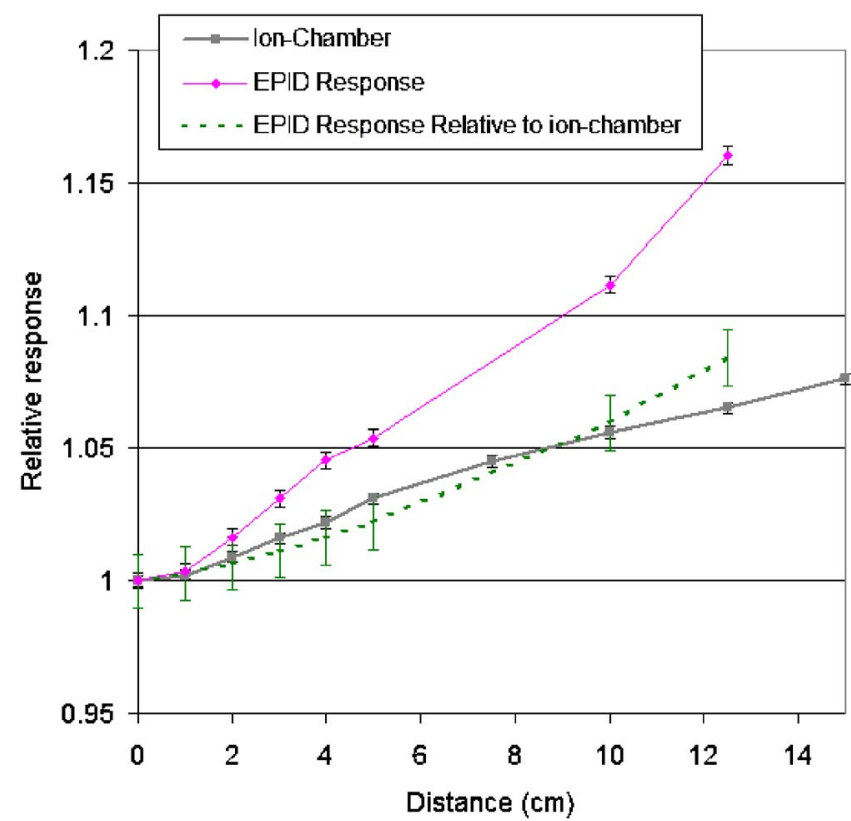

FIG. 4. EPID response for open beam radiation with respect to off-axis distance relative to ion chamber. The EPID response for the open 10 $\times 10 \mathrm{~cm}^{2}$ fields is compared to the measured ion-chamber dose. The response of the EPID relative to the ion chamber is also shown as the quadratic curve fit.

This is close to 1 within experimental uncertainty, suggesting that the MLC filtration results in a beam energy that is relatively homogeneous with off-axis distance.

The results for the EPID response to the open 10 $\times 10 \mathrm{~cm}^{2}$ field with off-axis distance is shown in Fig. 4. The response increases significantly with off-axis distance. These results are very similar to that previously measured with a $10 \times 25 \mathrm{~cm}^{2}$ field, ${ }^{19}$ with the response of the EPID relative to ion chamber at $12.5 \mathrm{~cm}$ off axis here being 1.09 , as previously measured.

\section{III.C. Effect of copper filters on the EPID energy- dependent response}

The effect of the copper filtration on the EPID response at the central axis is shown in Fig. 5. These results show that the copper does reduce the EPID response for the open beam; however, there is still a significant difference between the open and $\mathrm{MLC}_{\mathrm{tr}}$ response. The open beam EPID response is reduced by $\sim 4 \%$ for the $1 \mathrm{~cm} \mathrm{Cu}$ and $6 \%$ for the $1.6 \mathrm{~cm}$ $\mathrm{Cu}$. Although the $1.6 \mathrm{~cm}$ of $\mathrm{Cu}$ is equivalent to approximately $13 \mathrm{~cm}$ of water, this is much less than the effective thickness of the $6 \mathrm{~cm}$ thick tungsten MLC leaves, which are the equivalent of $116 \mathrm{~cm}$ of water. The EPID response to the $\mathrm{MLC}_{\mathrm{tr}}$ beam is not significantly affected by the $\mathrm{Cu}$ as would be expected with an already heavily filtered beam. The 1 and $1.6 \mathrm{~cm}$ of copper also attenuated the beam to 0.69 and 0.56 of the open beam dose rate, respectively, as determined from ion-chamber measurements.

Figure 6 shows the results for $12.5 \mathrm{~cm}$ off axis. The effect of the $\mathrm{Cu}$ filter on EPID response to the open beam is greater here due to the softer beam off axis. The EPID response is

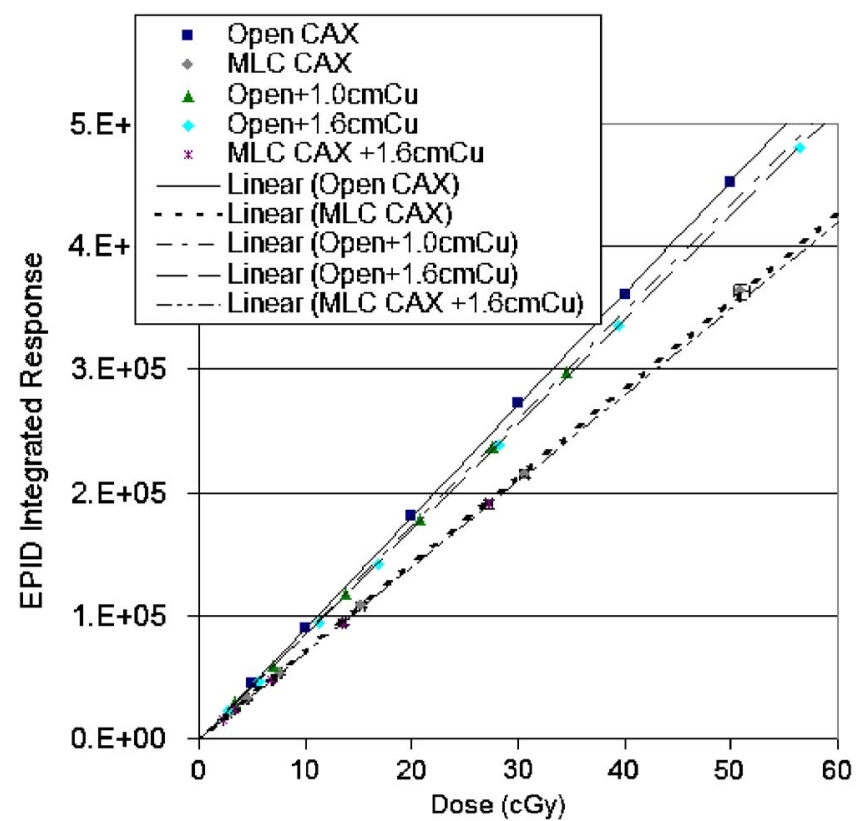

FIG. 5. The effect of copper filtration on the EPID response at the central axis. The open and MLC transmitted EPID responses were compared with additional 1 and $1.6 \mathrm{~cm}$ thicknesses of copper to filter low energy photons.

reduced by $\sim 8 \%$ for the $1 \mathrm{~cm} \mathrm{Cu}$ and $11 \%$ for the $1.6 \mathrm{~cm}$. However, there still remains a further $16 \%$ reduction in response to the $\mathrm{MLC}_{\mathrm{tr}}$ beam.

\section{III.D. Sliding window dynamic MLC beams}

Table I gives the results for the central axis measurements for the uniform sliding window beams with increasing $\mathrm{MLC}_{\mathrm{tr}}$ dose component. These show that the EPID response reduces relative to ion chamber with increasing $\mathrm{MLC}_{\mathrm{tr}}$ dose fraction, due to the lower EPID response for this component (Fig. 1). The predicted change in EPID response based on the measured calibration factors in Sec. I.B is in excellent agreement with the change in measured EPID response. These were within $2.3 \%$ for up to $69 \%$ of the dose delivered through the MLC, while the EPID response reduced by to $13 \%$ relative to ion chamber. These results suggest that by applying sepa-

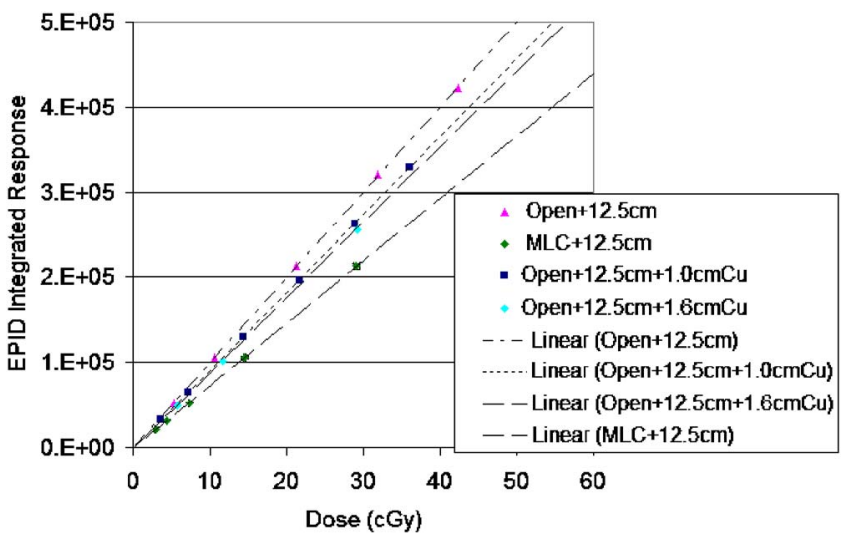

FIG. 6. The effect of copper filtration on the EPID response at $12.5 \mathrm{~cm}$ off axis. 


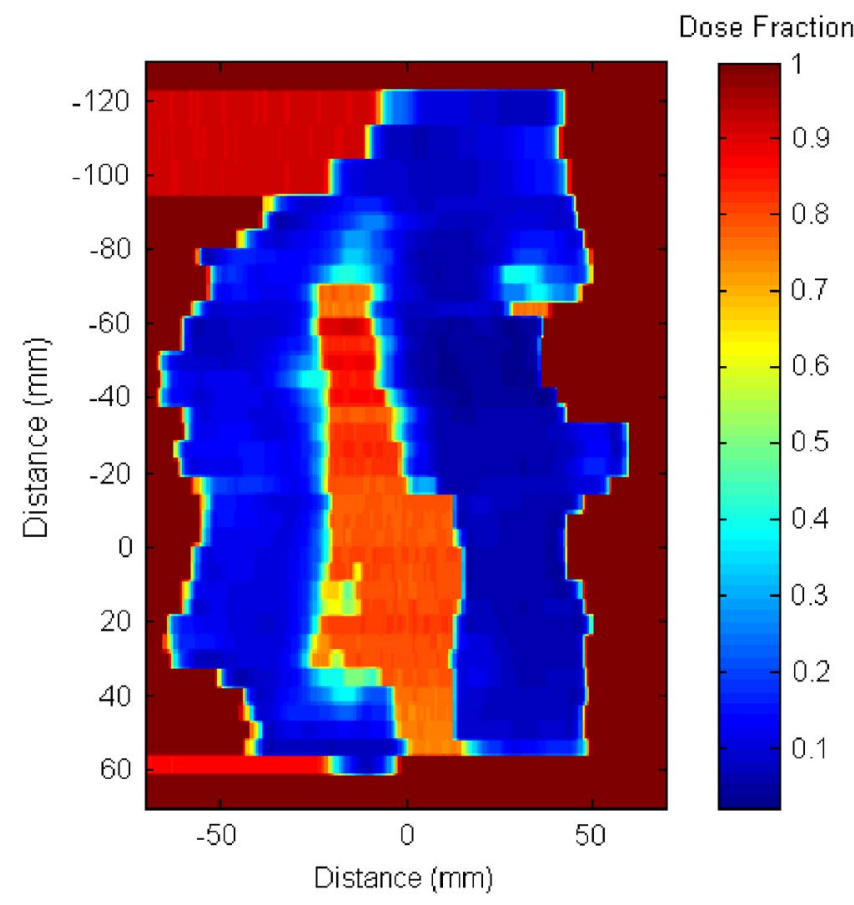

FIG. 7. Estimated fraction of delivered dose due to MLC transmission for the IMRT field. This was calculated from the dynamic MLC file.

rate EPID calibration factors to the open and MLCtransmitted components of an IMRT beam that improvements in EPID dosimetry will be achieved.

\section{III.E. Correction of energy-dependent response for IMRT beams}

Figure 7 shows the component of the primary dose delivered due to MLC-transmitted radiation for the clinical IMRT field. The $\mathrm{MLC}_{\mathrm{tr}}$ component is significant in this case in the center of the field. Figure 8 shows the percentage relative difference of the EPID image calibrated using the separate dose components [Eq. (5)], and the EPID image calibrated with the open field calibration matrix only. This shows that the errors introduced by assuming a single open field calibration are large, with a nearly $30 \%$ underprediction of the dose in the high MLC transmission regions. The dose in the high MLC transmission region near the central axis was approximately $20 \%$ of the maximum dose, showing that these regions are not necessarily very low in dose. Results are shown for the clinical prostate field in Fig. 9 for the MLCtransmitted dose fraction and in Fig. 10 for the percentage difference between the two calibration methods. In this case very little of the delivered dose within the field aperture is due to MLC leakage, and the errors in calibrating with a single matrix are much smaller, generally less than $1.5 \%$ within the field but higher outside the field.

\section{DISCUSSION}

The reduction in response of the EPID to MLCtransmitted radiation is due to the filtering of low energy photon component of the beam by the MLC. The EPID has a

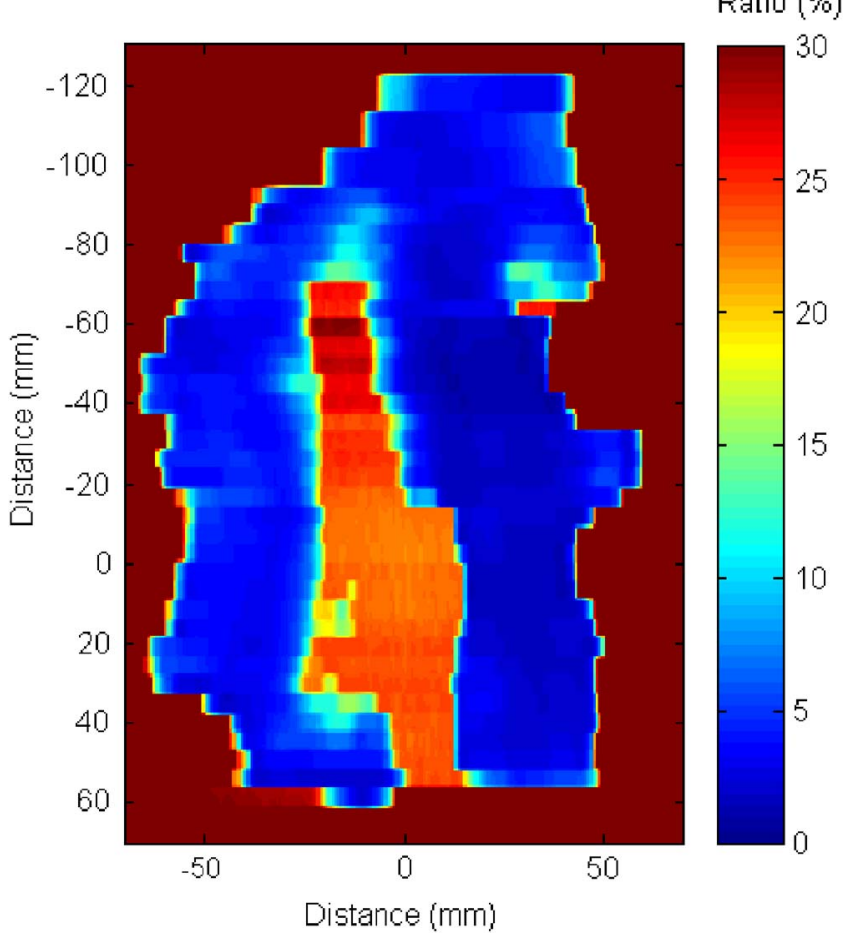

FIG. 8. The percentage difference between (1) The EPID calibrated with separate radially dependent calibration matrices for the open and MLCtransmitted components of the IMRT beam, and (2) The EPID calibrated with the open field radially dependent calibration matrix applied to both dose components of the beam.

higher response to the open beam when these photons are present due to the high atomic number of the phosphor layer. Charged particle interactions in the phosphor layer can occur from electrons produced by photon interactions in the copper buildup plate, or by electrons generated from interactions directly in the phosphor layer. The mass attenuation coefficient of gadolinium oxysulfide is relatively constant above $1 \mathrm{MeV}$. However below $1 \mathrm{MeV}$ it rapidly rises to be over

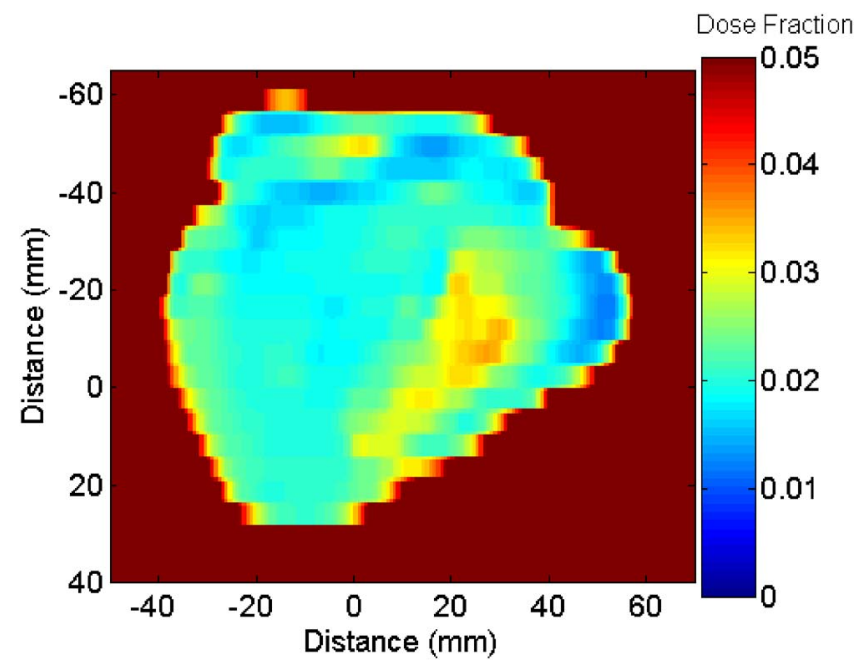

FIG. 9. The fraction of dose delivered due to MLC transmission for a prostate IMRT field. 


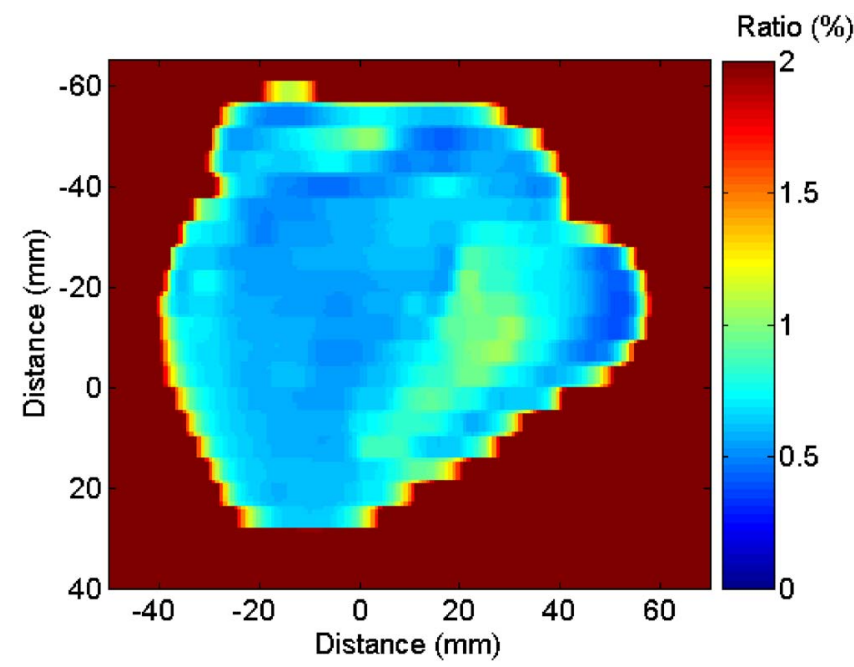

FIG. 10. The percentage difference between the EPID calibrated with the separate factors for open and MLC-transmitted dose components and the EPID calibrated with a single calibration matrix applied to both components.

three orders of magnitude higher at $10 \mathrm{keV}$ than at $1 \mathrm{MeV} .^{23}$ The EPID does not exhibit the significant change in response with off-axis distance to MLC-transmitted radiation that is apparent with open beam radiation.

The $\mathrm{Cu}$ filter was not very effective at reducing the difference in EPID response at central axis for open and MLCtransmitted beams. The $1.6 \mathrm{~cm} \mathrm{Cu}$ filter does reduce the EPID open field response relative to ion chamber at $12.5 \mathrm{~cm}$ off axis suggesting that a sufficient thickness of $\mathrm{Cu}$ would eventually eliminate the EPID open field energy-dependent response. However, very large thicknesses would be required, with corresponding large weight making this impractical. The $\mathrm{Cu}$ also modifies the beam profile significantly making comparison with the treatment planning system problematic.

The use of open field calibration factors for IMRT beams will introduce errors in IMRT dosimetry with EPIDs that increase with the MLC-transmitted component of the dose. ${ }^{22}$ As shown with the clinical IMRT field, these components can be significant for some fields depending on the clinical situation, and the errors in EPID dosimetry can be large. In conventional FF-correction EPID dosimetry, the FF acts as an open beam off-axis energy-dependent response correction, as the FF contains the EPID off-axis energy-dependent response to the large $\left(\sim 40 \times 30 \mathrm{~cm}^{2}\right) \mathrm{FF}$ calibration field (it also removes the beam profile from the acquired image). However this does not account for the difference in EPID response to the MLC-transmitted dose component both at central axis and with off-axis distance.

It is not known whether the result here would be affected by backscatter from the EPID housing. While the same field size was used for both open and MLC transmitted beams, as the energy spectrum incident on the EPID will be different, potentially the backscatter could also change. This could potentially be investigated by removal of the EPID from the support arm and comparing images for both types of beams. The relative differences in the open and MLC-transmitted
EPID response with and without the support arm would show whether the backscatter varies for these beams. However this would require the availability of a factory EPID to perform this experiment. ${ }^{8}$

Ghosting with the Varian EPID has been measured by McDermott et al. ${ }^{7}$ They found an underresponse of $\sim 5 \%$ at $5 \mathrm{MU}$ compared with $1000 \mathrm{MU}$. This reduced to less than $1.5 \%$ for $20 \mathrm{MU}$ and higher. The EPID response was not corrected in this paper for the effect of ghosting. We have measured the underresponse of our EPID as less than 3\% for $5 \mathrm{MU}$ and less than $1 \%$ for $10 \mathrm{MU}$ compared with $100 \mathrm{MU}$. These small reductions in response will not significantly affect the measured calibration factors, which were obtained by least squares fitting to EPID response data measured from 5 to $200 \mathrm{MU}$ for open beams.

In this work, the EPID image was calibrated to remove the change in response of the EPID relative to ion chamber for IMRT beams. Li et al. ${ }^{22}$ used Monte Carlo methods to determine EPID dose-deposition kernels for open and MLCtransmitted radiation as a function of radial distance. They found the ratio of the EPID kernel peaks (response) for the open beam relative to the MLC-transmitted beam at central axis was $\sim 1.3$. This is very similar to the response difference measured experimentally in this work. Their method however uses the separate kernels for open and MLC-transmitted dose components to predict the EPID response to the IMRT beam, which can then be compared to the measured EPID image, whereas the method developed here "calibrates out" the energy-dependent response. Their method also accounts for differences in the dose deposition kernel of open and MLC-transmitted components.

The calibration procedure does not correct for differences between ion chamber in water measurements and EPID response due to scatter differences. To account for these differences the EPID image could be compared to an EPID prediction model that uses a kernel to predict EPID scatter but does not incorporate energy-dependent response of the EPID. ${ }^{11}$ Or alternatively the EPID image calibrated with this method could be converted to a planar dose map in water using deconvolution/convolution techniques ${ }^{12}$ and compared with the treatment planning system dose to water calculation. A third approach is that the measured calibration factors could be incorporated into a prediction model to include the response of the EPID to beam energy variation, in a similar model to that used by $\mathrm{Li}$ et al. ${ }^{22}$ The prediction would then be compared directly to the PSM corrected EPID image. These was not done in this work as the purpose here was to isolate and investigate the effect of EPID energy-dependent response.

\section{CONCLUSIONS}

The difference in response of an amorphous silicon EPID to the open and MLC-transmitted beam components of IMRT beams was investigated. The response of the EPID to MLC-transmitted radiation was lower than for open beams due to the filtering of low energy photons by the MLC. The response of the EPID relative to ion chamber with off-axis 
distance was uniform for the MLC-transmitted beam whereas it increased markedly for open beam radiation. Copper filtering was not effective in reducing this difference in response. An EPID calibration method was developed for IMRT beams that uses the component of open and MLC transmitted dose to an EPID pixel and applies separate radially dependent calibration matrices to each component.

\footnotetext{
a) Author to whom correspondence should be addressed. Electronic mail: peter.greer@mater.health.nsw.gov.au. Telephone: 6124921 1510; Fax: 61249211138

${ }^{1}$ P. Munro and D. C. Bouius, "X-ray quantum limited portal imaging using amorphous silicon flat-panel arrays," Med. Phys. 25, 689-702 (1998).

${ }^{2}$ Y. El-Mohri, L. E. Antonuk, J. Yorkston, K. W. Jee, M. Maolinbay, K. L. Lam, and J. H. Siewerdsen, "Relative dosimetry using active matrix flatpanel imager (AMFPI) technology," Med. Phys. 26, 1530-1541 (1999). ${ }^{3}$ P. B. Greer and C. C. Popescu, "Dosimetric properties of an amorphous silicon electronic portal imaging device for verification of dynamic intensity modulated radiation therapy," Med. Phys. 30, 1618-1627 (2003).

${ }^{4}$ L. N. McDermott, R. J. W. Louwe, J.-J. Sonke, M. B. van Herk, and B. J. Mijnheer, "Dose-response and ghosting effects of an amorphous silicon electronic portal imaging device," Med. Phys. 31, 285-295 (2004).

${ }^{5}$ G. J. Budgell, Q. Zhang, R. J. Trouncer, and R. I. Mackay, "Improving IMRT quality control efficiency using an amorphous silicon electronic portal imager," Med. Phys. 32, 3267-3278 (2005).

${ }^{6} \mathrm{P}$. Winkler, A. Hefner, and D. Georg, "Dose-response characteristics of an amorphous silicon EPID,” Med. Phys. 32, 3095-3105 (2005).

${ }^{7}$ L. N. McDermott, S. M. J. J. G Nijsten, J.-J. Sonke, M. Partridge, M. van Herk, and B. J. Mijnheer, "Comparison of ghosting effects for three commercial $a$-Si EPIDs,” Med. Phys. 33, 2448-2451 (2006).

${ }^{8}$ L. Ko, J. O. Kim, and J. V. Siebers, "Investigation of the optimal backscatter for an aSi electronic portal imaging device," Phys. Med. Biol. 49, 1723-1738 (2004).

${ }^{9}$ R. J. W. Louwe et al., "The long-term stability of amorphous silicon flat panel imaging devices for dosimetry purposes," Med. Phys. 31, 29892995 (2004)

${ }^{10}$ J. Chang and C. C. Ling, "Using the frame-averaging of aS500 EPID for
} IMRT verification,” J. Appl. Clin. Med. Phys. 4, 287-299 (2003).
${ }^{11}$ A. van Esch, T. Depuydt, and D. P. Huyskens, "The use of an aSi-based EPID for routine absolute dosimetric per-treatment verification of dynamic IMRT fields," Radiother. Oncol. 71, 223-234 (2004).

${ }^{12}$ B. Warkentin, S. Steciw, S. Rathee, and B. G. Fallone, "Dosimetric IMRT verification with a flat-panel EPID," Med. Phys. 30, 3143-3155 (2003).

${ }^{13}$ J. V. Siebers, J. O. Kim, L. Ko, P. J. Keall, and R. Mohan, "Monte Carlo computation of dosimetric amorphous silicon electronic portal images," Med. Phys. 31, 2135-2146 (2004).

${ }^{14}$ S. Steciw, B. Warkentin, S. Rathee, and B. G. Fallone, "Threedimensional IMRT verification with flat-panel EPID," Med. Phys. 32, 600-612 (2005).

${ }^{15}$ G. Nicolini, A. Fogliata, E. Vanetti, A. Clivio, and L. Cozzi, "GLAaS: An absolute dose calibration algorithm for an amorphous silicon portal imager. Applications to IMRT verifications," Med. Phys. 33, 2389-2851 (2006).

${ }^{16}$ M. Wendling, R. J. W. Louwe, L. McDermott, J.-J. Sonke, M. Van Herk, and B. J. Mijnheer, "Accurate two-dimensional IMRT verification using a back-projection EPID dosimetry method," Med. Phys. 33, 259-273 (2006).

${ }^{17}$ B. M. C. McCurdy, K. B. Luchka, and S. Pistorius, "Dosimetric investigation and portal dose image prediction using an amorphous silicon electronic portal imaging device," Med. Phys. 28, 911-924 (2001).

${ }^{18}$ C. Kirkby and R. Sloboda, "Consequences of the energy-dependent response of an a-Si EPID and implications for dosimetric calibration," Med. Phys. 32, 2649-2658 (2005).

${ }^{19} \mathrm{P}$. B. Greer, "Correction of pixel sensitivity variation and off-axis response for amorphous silicon EPID dosimetry," Med. Phys. 32, 35583568 (2005).

${ }^{20}$ L. Parent, J. Seco, P. M. Evans, A. Fielding, and D. R. Dance, "Monte Carlo modeling of $a$-Si EPID response: The effect of spectral variations with field size and position," Med. Phys. 33, 4527-4540 (2006).

${ }^{21}$ P. Vial, P. B. Greer, L. Oliver, and L. C. Baldock, "An experimental investigation into the radiation field offset of a dynamic multileaf collimator," Phys. Med. Biol. 51, 5517-5538 (2006).

${ }^{22}$ W. Li, J. V. Siebers, and J. A. Moore, "Using fluence separation to account for energy spectra dependence in computing dosimetric $a$-Si EPID images for IMRT fields," Med. Phys. 33, 4468-4480 (2006).

${ }^{23}$ National Institute of Standards and Technology, Physical Reference Data, Tables of X-Ray Mass Attenuation Coefficients and Mass EnergyAbsorption Coefficients. 